cardiogenic shock at presentation and 2 had out of hospital cardiac arrest. Hypertension $(n=19)$, smoking history $(n=18)$, hypercholesterolemia $(n=16)$, diabetes $(n=6)$ and chronic kidney disease $(n=6$ with eGFR $<60)$ were frequently present. Aspirin was given to 20/21 patients and clopidogrel, ticagrelor and prasugrel to 10, 9 and 3 patients respectively. Heparin was used in all patients, glycoprotein (GP) IIBIIIA inhibitor in 10 and bivalirudin in 2 .

Radial access was used in 14 and femoral in 7. Initial TIMI flow grade was $0,1,2$ and 3 in $11,2,1$ and 7 patients respectively. The target vessel was the RCA in 14, Cx in 4, LMS in 2 and LAD in 1. All were severely calcified with visible thrombus in 13/21. The lesion length was 36(19) $\mathrm{mm}$ (range 12-72 mm). The vessel diameter was $2.5-2.99 \mathrm{~mm}$ in $5,3-3.49 \mathrm{~mm}$ in 10 and $3.5-4.0 \mathrm{~mm}$ in 6 . RA was used because of anticipated difficulty with conventional PCI in $7 / 21$ and uncrossable/unexpandable lesions in 14/21. The number of burrs used was $1.33(0.48)$ and the final burr was 1.25 , $1.5,1.75$ and $2 \mathrm{~mm}$ in $4,11,4$ and 1 patient respectively. The burr-to-artery ratio was $<0.4$ in 5 and $0.4-0.6$ in 16 . The final procedure was DES in 17, BMS in 3 and POBA followed by $\mathrm{CABG}$ in 1 . A temporary pacemaker was used in 3 cases and intra-aortic balloon pump in 3 cases. There were no perforations, no tamponade and no vascular complications.

The median(range) pain-to-PCI time was $180 \mathrm{~min}$ (114544); door-to-PCI time $49 \mathrm{~min}(21-186,14$ patients $<60 \mathrm{~min}$ ) and procedure time was $119 \mathrm{~min}(66-175)$. Procedural difficulty caused door-to-PCI $>60 \mathrm{~min}$ in $5 / 7$ patients while medical/logistic reasons contributed in the other 2.1 patient underwent CT scanning prior to PCI; 1 underwent an urgent MDT discussion before PCI. Final flow was TIMI 3 in $19 / 21$ and TIMI 2 in $2 / 21$ cases. The procedure was complicated by 1 event of distal embolization. There was 1 event of peri-procedural cerebral infarction. 20/21 patients survived to hospital discharge.

Conclusions Our study suggests that RA PPCI can be performed safely in a small group of predominantly elderly, complex patients in whom conventional techniques are inadequate or unsuccessful. It is associated with a relatively long procedure time but we did not experience a marked increase in no-reflow.

\section{COMPLICATION RATE OF CORONARY ANGIOGRAPHY IN PATIENTS WITH PRIOR CORONARY ARTERY BYPASS GRAFTING}

Perin Celebi, Mohamad Fahed Barakat, Omar Chehab, Nicholas Aveyard*, Tim Lockie. Royal Free Hospital NHS Trust

\subsection{6/heartjnl-2017-311726.28}

Background When consenting patients for elective coronary angiography, a risk of major complications such as death, stroke or myocardial infarction is quoted at $1-2$ in 1000 . However, what is the risk for patients who have had a prior coronary artery bypass graft surgery (CABG)? It is believed to be higher than the basic complication rate due to a lengthier, more complex procedure. We aim to estimate the complication rate in this cohort of patients, and identify factors that increase this risk.

Methods Between April 2008 and August 2016, a cohort of 681 consecutive patients undergoing elective coronary angiography with a history of CABG was collected retrospectively from a district general hospital in London. The primary outcome was Major Adverse Cardiac or Cerebrovascular Events (MACCE-death, stroke, myocardial infarction). Our secondary outcome was vascular complications (pseudoaneurysm, ischaemic limb, retroperitoneal bleed, significant haematoma requiring transfusion). The complication rates were calculated and then a backward logistic regression model constructed to identify the strongest predictor variables.

Results The 681 patient cohort consisted of 566 (83\%) male patients and the mean age was 72 years $( \pm 9)$. The majority of cases $(626,92 \%)$ were performed transfemorally. The median procedural time was $45 \mathrm{mins}$ (IQR: 40-55). The overall MACCE rate was 23 per 1000. Vascular complications were observed at 5.9 per 1000 . Using the regression model the single strongest predictor of MACE and vascular complications is procedural time. For every additional $10 \mathrm{~min}$ there is a 1.261 fold increase in risk of MACE (95\% CI: 1.111-1.596, $\mathrm{p}=0.042$ ), and a 1.826 fold increase in risk of vascular complications (95\% CI: 1.309-2.137, $\mathrm{p}=0.001$ ).

Conclusion The findings of this study estimates the MACE and vascular complication rates in patients with a previous CABG at 23 per 1000 and 5.9 per 1000 respectively. It demonstrates that procedural time is a strongest predictor of either of these complications.

\section{ASSESSING LONG TERM TRANSCATHETER AORTIC VALVE IMPLANTATION (TAVI) FUNCTION BY CLINCAL ECHOCARDIOGRAPHY AT A SINGLE UK TAVI CENTRE}

${ }^{1}$ Akhlaque Uddin, ${ }^{2}$ Ken-win To, ${ }^{1}$ Imogen Woods, ${ }^{1}$ Sofia Kassou*, ${ }^{1}$ Elved Roberts, ${ }^{1}$ David Adlam, ${ }^{1}$ Derek Chin, ${ }^{1}$ Jan Kovac. ${ }^{1} \mathrm{NHS} ;{ }^{2}$

\subsection{6/heartinl-2017-311726.29}

Background Recent concerns regarding long term transcatheter aortic valve implantation (TAVI) prosthesis failure have been raised. Studies with long term failure outcomes are limited.

Objective A cross sectional study to assess TAVI prosthesis function after more than 5 years implantation.

Method All successful TAVI procedures at a single high volume centre between January 2007 to December 2012 were included. The most recent clinical transthoracic study assessing for prosthetic function performed by accredited cardiac sonographers and physicians were reviewed. The duration of follow up scan was categorised to early ( $<12$ months), medium term (12 to 48 months) and long term (>48 months). Patient mortality data was recorded.

Results 212 TAVI procedures were successfully implanted with follow up ranging from 4 to 9 years. The characteristics are similar to the first generation TAVI population with predominant use of a femoral approach and a self-expanding device. 4 $(1.8 \%)$ patients died pre-discharge. 30 day mortality was, $3.3 \%$. 1st, 2nd, 3rd, 4th years mortality were $11.9 \%, 7.0 \%$, $13.9 \%, 16.9 \%$ respectively. Survival at 5 years was $53.3 \%$.

There were fewer patients with follow-up scans beyond 48 months. Cross sectional comparison did not show a change in valve gradient. Mild paravalvular regurgitation was present consistently. There was no evidence of late aortic stenosis or severe AR.

Conclusion The medium to long term 1st generation TAVI valve function is promising, but long term follow up will be difficult due to high mortality in the earlier implantations. 